\title{
Experimental Study on Low-Grade Waste Heat Recovery with Thermoelectric Generators
}

\author{
Abhishek Khanchi ${ }^{1 *}$, Mani Kanwar Singh' ${ }^{1}$ Satbir S. Sehgal' and Harkirat Sandhu ${ }^{2}$ \\ 'Department of Mechanical Engineering, Chandigarh University, Gharuan, Mohali - 140413, Punjab, India; \\ abhishek110793@gmail.com, moneykanwar@gmail.com,drsatbirsehgal@gmail.com \\ 2Department of Chemical Engineering, Chandigarh University, Gharuan, Mohali - 140413, Punjab, India; \\ harkiratsandhu86@gmail.com
}

\begin{abstract}
Low-grade waste heat recovery has emerged as a need for both efficient and sustainable approach. Till now conventional systems have failed to provide any assistance in harnessing and efficient conversion of low-grade to medium grade heat because of their huge sizes, high investment in land and equipment, complexity in design and scarcity in their availability. Researchers are now trying to solve these problems with the use of thermoelectric generators. These solid-state semiconductor devices can convert heat fluxes into DC output by maintaining a certain temperature gradient across its surfaces. Depending on the device and its rating power output increases with the rise in temp gradient. Objective: To identify errors with TEG module and if possible to perform investigation on it. Method/Statistical Analysis: In this method, two different positions were used. Firstly, one heat input surface was upside (hot side up) and second with heat input from below (Hot side down). This change in position will affect the convective motion of trapped air molecules and effect (if any) could be noticed. Findings: For hot side down position, there was an increment of $0.22 \%$ voltage output, $0.44 \%$ more power output and $0.521 \%$ increment in See beck coefficient observed in comparison with hot side up position.
\end{abstract}

Keywords: Low-grade Waste Heat Recovery, Semiconductor Device, Solar Pond, Sustainable, Temperature Gradient, Thermoelectric Generator

\section{Introduction}

Waste heat management for efficient and sustainable development has emerged as one of the most heated topic at present. Statistical reports regarding environmental degradation has propelled researchers and engineers around the world to tackle these problems head on and to find a viable- sustainable solution for this global crisis. Usually industrial waste recovery is done using ranking cycle or the waste heat is consumed for other purposes via heat transfer using heat exchangers. But, the scope of this system is very limited to large-scale industries to few medium-scale industries only, because of high investment on infrastructure, land requirement, machinery, qualified workers. Even after all these expenses the output efficiency is only $20-25 \%$.
Moreover, this percentage conversion reduces even more for medium to low-grade waste heat.

This is where thermoelectric generators come to our rescue. A thermoelectric device or generator is a solidstate semiconductor device which works on Seebeck principle and has inherent characteristic to convert heat flux directly into DC output $t^{-\underline{-5}}$. Another positive feature of these devices is their sleek, slim and compact physical design which makes it extremely versatile in its employment in almost every field ${ }^{6}$. Over the years, various researchers have fabricated different and innovative systems to harness waste heat from vehicle exhaust, cooking apparatus, industrial burners, solar ponds, geothermal energy source etc ${ }^{\underline{z}}$. Most of the setups involve use of multiple units in parallel and series connections to get certain output amplitude of voltage or current ${ }^{3}$. These fabricated

${ }^{*}$ Author for correspondence 
power units make no sound, produces no harmful byproduct, requires no maintenance, are highly reliable and are inexpensive to buy in large quantities ${ }^{3}$.

These positive traits have propelled researchers to design and develop various kinds of low-grade waste heat recovery systems. In ${ }^{1}$ fabricated TEG based generator for waste heat recovery from water heater using natural gas as fuel. This generator unit had 60 individual TEG units. $\mathrm{In}^{2}$ conceptualized low-grade waste heat recovery system using a system of heat pipe with TEG unit sandwiched between heat transfer surfaces. Finns were used for heat addition and removal. Theoretical analysis showed the system was capable of generation 10.39 watts from $1.345 \mathrm{~kW}$ of primary heat. $\operatorname{In}^{3}$ fabricated a generator unit comprising of 600 individual TEG units. Here main heat source was geothermal energy. Power generating capacity of generator was $1 \mathrm{~kW}$. $\mathrm{In}^{4}$ prepared low-grade heat harnessing and conversion system with solar ponds as heat source. Temperature difference between upper convective layer and lower convective layer was utilized.

$\operatorname{In}^{6}$ performed theoretical analysis to check efficiency of TEG-Organic Rankine Cycle combined together. R123 refrigerant was used as working fluid system. $\mathrm{In}^{7}$ theorized a conceptual model for harnessing het rays of sun and for cooling Phase change material (paraffin wax) was used. Further enhancement was performed by using principle of thermosiphon for removal of waste heat from PCM storage container.

Primary objective of this experimental research work is to find flaws with TEG unit, which is blocking it to attain higher efficiency. It is possible that the gaps present inbetween P-N junctions of TEG are leaking heat from it. It is also possible that this leakage is also affecting its working efficiency too. For validating this, here the direction of heat transfer to the TEG unit is being changed (setup one with hot side upwards and another one with hot side down) with other variables kept same or unchanged. This variation will lead to some change in heat transfer rate (if heat leakage effect is present) and variation in power output (if heat leakage does effect output). These are some of the questions that can be answered by this experimental study.

\section{Materials and Method}

Figure 1 shows schematic diagram of the setup. Heat addition and removal from the TEG unit was achieved with the help of heat exchanger fabricated using quarter inch refrigeration purpose copper tubes integrated in CPVC and PVC pipes. Further, copper pipes were enveloped by a 42-gauge copper sheet for uniform addition and removal of heat as shown in Figure 2. Final setup of TEG unit with heat exchangers is shown in Figure 3. In this experiment regular tap water was used as working fluid. For cold water circuit or heat removal circuit PVC pipes were used and for hot water circuit CPVC pipes were used. Reason for choosing polymer pipes was to avoid any heat losses during motion of fluid from and to the fluid reservoirs.

For temperature measurement K-Type thermocouples with digital indicator (Model: 50338 manufactured by Cooper Atkins) was used. Response time for thermocouple is 1 second, with accuracy of $\pm 0.20 \mathrm{C}$ and temperature range of $0^{\circ} \mathrm{C}-480^{\circ} \mathrm{C}$.

\section{Results and Discussions}

For various calculations and finding quantitative aspects following mathematical expressions were used:

Seebeck Coefficient $=\left(\mathrm{V}_{\mathrm{od}} / \mathrm{dt}\right)$

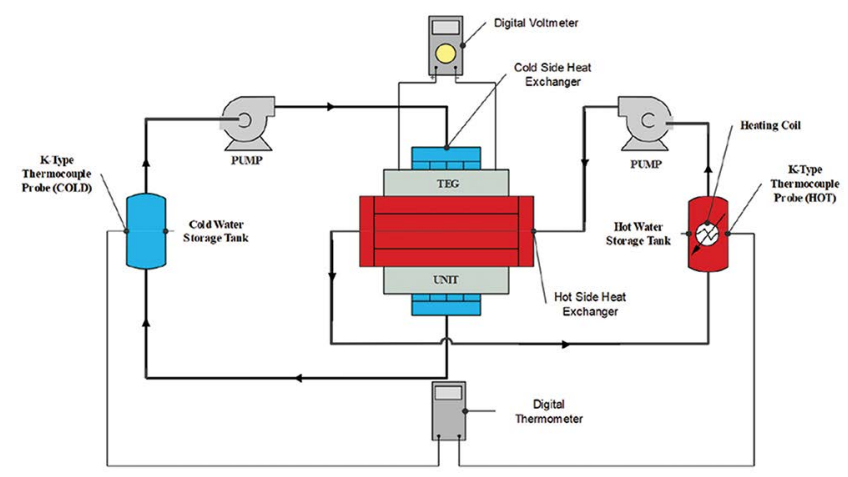

Figure 1. Schematic diagram of experimental setup.

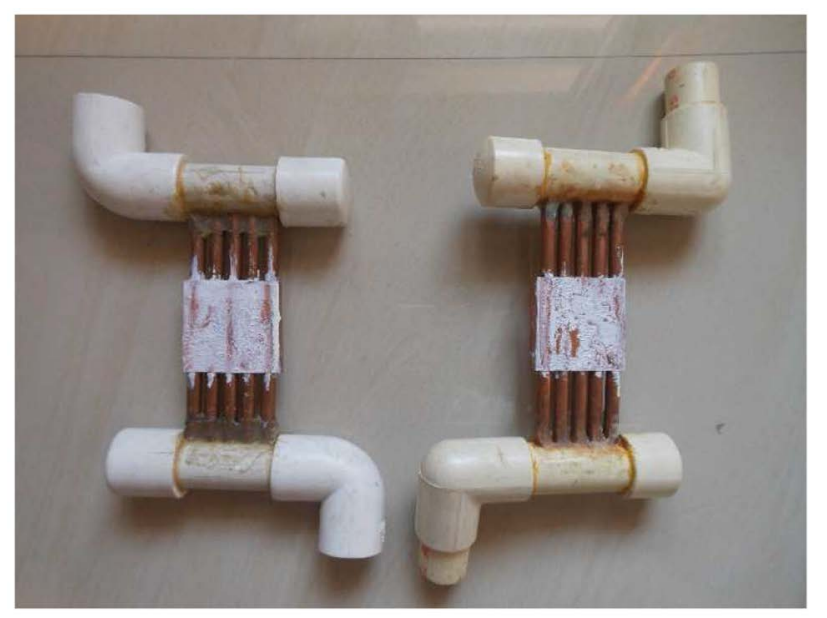

Figure 2. Heat exchangers with heating surface. 


$$
\begin{aligned}
& \text { Conversion Unit, } \mathrm{M}=\left[1+\left[(\mathrm{Z} / 2) *\left(\mathrm{~T}_{\mathrm{H}}-\mathrm{T}_{\mathrm{C}}\right)\right]\right]^{1 / 2} \\
& \text { Power Output, } \mathrm{P}=\mathrm{I}^{2 *} \mathrm{R} \\
& \mathrm{N}_{\text {ideal }} \quad=\text { Ideal Efficiency }(\%)=\left(\left(\mathrm{T}_{\mathrm{H}}-\right.\right. \\
& \left.\left.\mathrm{T}_{\mathrm{C}}\right) / \mathrm{T}_{\mathrm{C}}\right) \times\left[(\mathrm{M}-1) /\left(\mathrm{M}+\left(\mathrm{T}_{\mathrm{C}}\right.\right.\right. \\
& \left.\left.\left(\mathrm{T}_{\mathrm{C}}\right)\right)\right] \\
& \mathrm{V}_{\mathrm{OC}} \quad=\text { Open Circuit Voltage, (in Volts) } \\
& \mathrm{dt} \quad=\text { Temp Difference, (in degree } \\
& \text { Celsius) } \\
& \mathrm{Z} \quad=\text { Figure of Merit, value calcu- } \\
& \text { lated to be }=2119 \mathrm{~K}-1 \text {, } \\
& \mathrm{T}_{\mathrm{H}} \quad=\text { Temp of hot side of TEG, } \\
& \mathrm{T}_{\mathrm{C}} \quad=\text { Temp of cold side of TEG, } \\
& \text { I = Output current of TEG, (in } \\
& \text { Amps) } \\
& \mathrm{R}=\text { Internal resistance of TEG, (in } \\
& \text { ohms) }
\end{aligned}
$$

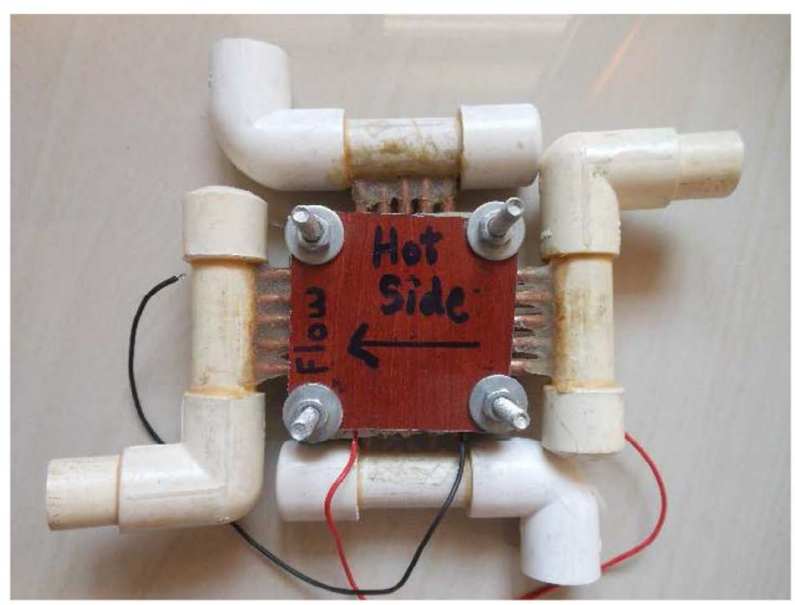

Figure 3. TEG test setup with hot side UP.

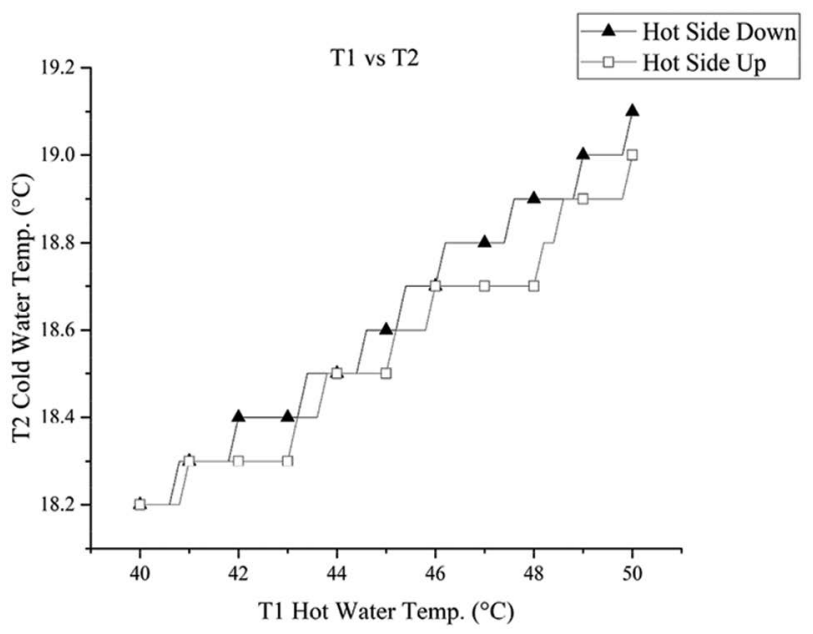

Figure 4. Hot water $\operatorname{Temp}\left(\mathrm{T}_{1}\right)$ Vs Cold Water $\operatorname{Temp}\left(\mathrm{T}_{2}\right)$.

\subsection{Rate of Heat Transfer}

Rate of heat transfer is the amount of heat transferred from point $A$ to point $B$ in unit time. Figure 4 shows Hot water temperature Vs Cold water temperature graph. In this case as we change the position of heat input of hot side of TEG, a variation in heat transfer rate can be clearly observed. Heat transfer rate for hot side down is more and uniform, while for hot side up its less and non-uniform. This change in heat transfer rate indicates heat leakages within TEG unit.

\subsection{Effect of Heat Leakages on Voltage and Power Output}

For a TEG unit its output in terms of power and voltage is directly proportional to the temperature gradient maintained across its surfaces. In simple terms more the temperature difference, more will be the power and voltage output of the TEG. Figures 5, 6 shows Temperature Gradient Vs Voltage and Power of TEG respectively. So, theoretically hot side UP should have better voltage and power output, as temperature gradient for hot side up is more throughout the experiment. But, it's exactly opposite here with hot side down setup has higher magnitude of power and voltage.

Reason: In down position air molecules trapped in-between surfaces transfers heat directly to upper surface and reducing any heating effect of P-N legs of TEG, while in hot side UP this effect is causing overheating of $\mathrm{P}-\mathrm{N}$ legs and reducing power output of TEG.

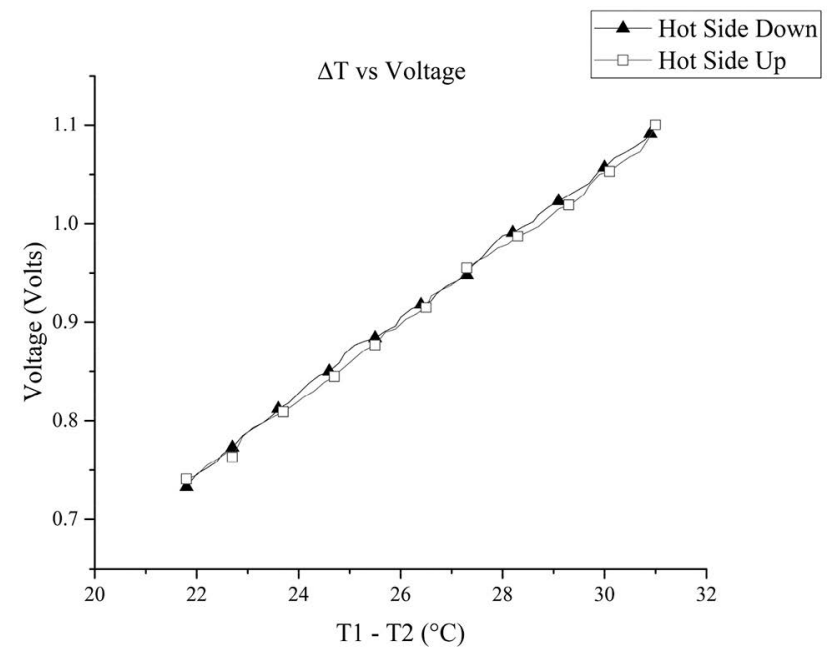

Figure 5. Temperature gradient vs voltage. 


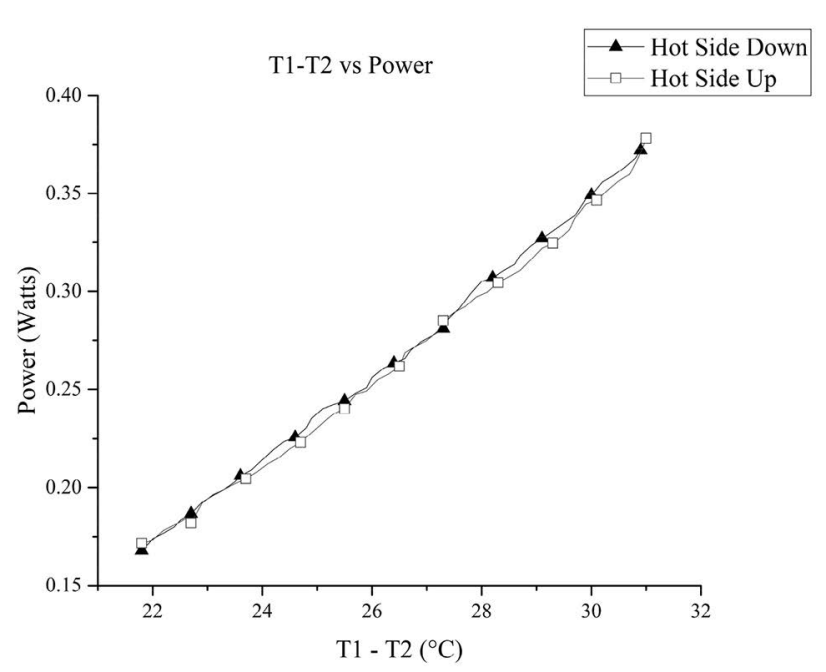

Figure 6. Temperature gradient vs power.

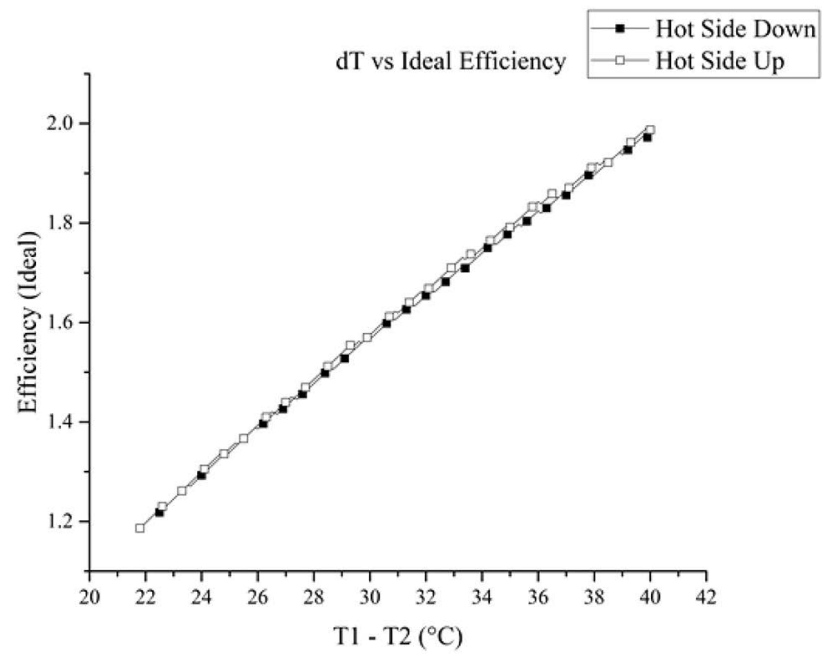

Figure 7. Temperature difference vs ideal efficiency.

\subsection{Effect on Ideal Efficiency}

Just like voltage and power, ideal efficiency is also directly or indirectly dependent on temperature gradient maintained across TEG's surfaces, which is higher in case of hot side upand hence mathematically, ideal efficiency of hot side up setup is higher. Figure 7 depicts Temperature Gradient vs ideal efficiency graph.

Results obtained from experimental analysis of TEG indicate a variation in theoretical and experimental reading. This experiment also indicates to the possibility of heat leakages and its negative impact on power output of TEG. Mathematically, $0.22 \%$ and $0.44 \%$ increase in voltage and power output was calculated in hot position down as compared to hot position up. This may be insignificant for single TEG unit but for a generator comprising of 600 or more units this could enhance its working efficiency exponentially.

\section{Conclusions}

From the experiments, following conclusion can be reported as:

1. Low-grade waste heat recovery using TEGs from sources like vehicle exhausts, solar ponds, water heaters, cooking apparatuses is absolutely feasible.

2. Heat leakages do occur from hot side of TEG and is instantaneous. Heat transfer rate turned out to be more for down position of TEG.

3. Change in position causes a change in direction of convection currents and hence change in heat transfer rate. For this experiment, hot side down position proves best for heat recovery.

4. Power output of TEG does get affected by heat leakages. But, overall primary effect cannot be calculated because of the absence of TEG module without heat leakage effect.

5. Output efficiency is directly proportional to the temperature gradient maintained across its surfaces.

\section{References}

1. Ding LC, Meyerheinrich N, Tan L. Thermoelectric power generation from waste heat of natural gas water heater. Energy Procedia. 2017; 1:1032-37. Crossref.

2. Remeli MF, Tan L, Date A. Simultaneous power generation and heat recovery using a heat pipe assisted thermoelectric generator system. Energy Conversion and Management. 2015; 91:110-19. Crossref.

3. Liu C, Chen P, Li K. A $1 \mathrm{KW}$ thermoelectric generator for low-temperature geothermal resources. Thirty-Ninth Workshops on Geothermal Reservoir Engineering Stanford University, Stanford, California, SGP-TR-202; 2014. p. 1-12.

4. Singh B, Remeli F, Oberoi A. Electrical power generation from low grade heat of salinity gradient solar pond using thermoelectric generators. Proceedings of the 52nd Annual Conference, Australian Solar Energy Society (Australian Solar Council); 2014.

5. Singh B, Varthani J, Remeli MF. Experimental investigation of power generation from salinity gradient solar pond using thermoelectric generators for renewable energy application. Applied Mechanics and Materials. 2013; 393:809-14. Crossref. 
6. Shu G, Zhao J, Tian H. Parametric and exergetic analysis of waste heat recovery system based on thermoelectric generator and organic Rankine cycle utilizing R123. Energy. 2012; 45(1):806-16. Crossref.
7. Tan L, Singh R, Date A. Thermal performance of two-phase closed thermosyphon in application of concentrated thermoelectric power generator using phase change material thermal storage. Frontiers in Heat Pipes; 2011. 